\title{
Distinct TIr4-expressing cell compartments control neutrophilic and eosinophilic airway inflammation
}

\author{
JW McAlees ${ }^{1}$, GS Whitehead ${ }^{2}$, ITW Harley ${ }^{1}$, M Cappelletti $^{1}$, CL Rewerts ${ }^{1}$, AM Holdcroft $^{1}$, S Divanovic $^{1}$, \\ M Wills-Karp ${ }^{1,5}$, FD Finkelman ${ }^{1,3,4}$, CL Karp ${ }^{1,6}$ and DN Cook ${ }^{2}$
}

Allergic asthma is a chronic, inflammatory lung disease. Some forms of allergic asthma are characterized by $\mathrm{T}$ helper type 2 (Th2)-driven eosinophilia, whereas others are distinguished by Th17-driven neutrophilia. Stimulation of Toll-like receptor 4 (TLR4) on hematopoietic and airway epithelial cells (AECs) contributes to the inflammatory response to lipopolysaccharide (LPS) and allergens, but the specific contribution of TLR4 in these cell compartments to airway inflammatory responses remains poorly understood. We used novel, conditionally mutant $T I r 4^{f l / f l}$ mice to define the relative contributions of AEC and hematopoietic cell TIr4 expression to LPS- and allergen-induced airway inflammation. We found that TIr4 expression by hematopoietic cells is critical for neutrophilic airway inflammation following LPS exposure and for Th17-driven neutrophilic responses to the house dust mite (HDM) lysates and ovalbumin (OVA). Conversely, TIr4 expression by AECs was found to be important for robust eosinophilic airway inflammation following sensitization and challenge with these same allergens. Thus, TIr4 expression by hematopoietic and airway epithelial cells controls distinct arms of the immune response to inhaled allergens.

\section{INTRODUCTION}

Allergic asthma is an increasingly prevalent disease that is characterized by chronic inflammation of the airway and reversible airway obstruction. Asthma is widely considered to stem from allergen-specific Thelper type 2 (Th2) responses that result in eosinophilic inflammation but, in many individuals, neutrophils are the predominant leukocytes in the airway. ${ }^{1}$ Several studies have suggested that neutrophilic forms of asthma arise from allergen-specific Th17 responses, ${ }^{2,3}$ and are characterized by airway hyperresponsiveness and resistance to glucocorticoid therapy. ${ }^{4-6}$ These findings have been replicated in mouse models of experimental allergic asthma. ${ }^{4,7}$ Defining the cellular and molecular events that promote Th2 and Th17 responses to inhaled allergens will likely be critical for developing novel, effective strategies targeting specific subtypes of asthma.

A large body of evidence suggests that many allergens trigger maladaptive immune responses by direct interactions with innate immune receptors such as Toll-like receptors (TLRs). ${ }^{8}$
The best-characterized TLR in this regard is TLR4 that signals in response to lipopolysaccharide (LPS), a membrane component of Gram-negative bacteria. ${ }^{9}$ However, the relationship between environmental LPS and asthma is complex. Whereas some epidemiologic studies have linked LPS exposure to an increased prevalence of asthma, ${ }^{10,11}$ other studies have suggested that exposure to LPS decreases the risk of developing allergic asthma. ${ }^{12}$ Mouse models of asthma have confirmed a role for LPS during allergic sensitization to experimental allergens, with very high doses of inhaled LPS triggering Th1 responses to ovalbumin (OVA), and lower doses promoting Th2 and Th17 responses. ${ }^{13,14}$ In addition to LPS, some bona fide allergens display structural and functional homology to components of the TLR4 receptor complex, and can directly trigger TLR4 signaling and consequent allergic sensitization. ${ }^{15}$ Despite this wealth of evidence supporting an important role for TLR4 in the development of allergic responses, the specific function of this receptor during allergic sensitization remains unclear. In particular, studies employing bone marrow chimera

\footnotetext{
${ }^{1}$ Division of Immunobiology, Cincinnati Children's Hospital Research Foundation, Cincinnati, Ohio, USA. ${ }^{2}$ Laboratory of Respiratory Biology, National Institutes of Environmental Health Sciences, National Institutes of Health, Department of Health and Human Services, Research Triangle Park, North Carolina, USA. ${ }^{3}$ Department of Medicine, Cincinnati Veterans Affairs Medical Center, Cincinnati, Ohio, USA. ${ }^{4}$ Department of Internal Medicine, Division of Allergy, Immunology and Rheumatology, University of Cincinnati School of Medicine, Cincinnati, Ohio, USA. ${ }^{5}$ Present address: Johns Hopkins Bloomberg School of Public Health, Baltimore, MD, USA and ${ }^{6}$ Bill \& Melinda Gates Foundation, Seattle, WA, USA. Correspondence: DN Cook (cookd@niehs.nih.gov)
} 
techniques have led to diverse conclusions regarding the relative contribution of different Tlr4-expressing cell populations to LPS or allergen responsiveness. ${ }^{16-18}$ Unfortunately, experiments of this type are confounded by the persistence of large numbers of host macrophages and dendritic cells after irradiation. ${ }^{19}$ Furthermore, although airway epithelial cells (AECs) are widely regarded as radioresistant, radiation induces profound transcriptional changes in these cells that have unknown effects on their function. ${ }^{20}$ Thus, although studies of irradiated bone marrow chimeric mice are useful, they have inherent limitations.

Here, we describe the creation of conditionally mutant Tlr4 mice that can be used to delete Tlr4 in distinct cell compartments without the complications associated with irradiation. Our studies revealed that Tlr4 expression by hematopoietic cells controls neutrophilic responses to inhaled LPS and allergens, whereas expression of this receptor by AECs is important for eosinophilic responses to inhaled allergens.

\section{RESULTS}

\section{Generation of mice lacking TIr4 expression by AECs or hematopoietic cells}

We generated conditionally mutant Tlr4 mice by flanking the third exon of Tlr4 with LoxP sites (Tlr $4^{f l f l}$ ) (Figure 1a). This strategy was chosen because germline mutant mice lacking the third exon are unresponsive to LPS. ${ }^{9}$ To inactivate Tlr4 in the airway epithelium, Tlr $4^{f l f l}$ mice were bred to animals in which expression of Cre recombinase is controlled by the Shh locus (Shh-Cre mice). ${ }^{21}$ Shh expression by the lung epithelium arises at E9.5 with full expression throughout the lung epithelium by E12.5. ${ }^{22,23}$ Thus, Cre-mediated genetic excisions are maintained in the progeny of these cells, including all pulmonary epithelial cell lineages. ${ }^{24}$ Furthermore, to inactivate TLR4 in the hematopoietic compartment, $T l r 4^{f l f l}$ mice were bred to transgenic animals expressing Cre under control of the HS21/45vav1 oncogene promoter (Vav1-Cre mice) ${ }^{25}$

Selective Tlr4 deletion in AECs of $T l r 4^{f l / f l}$ Shh-Cre mice, and in hematopoietic cells of Tlr $4^{f l f l}$ Vav1-Cre mice, was confirmed by PCR analysis of genomic DNA (Figure 1b) and by analysis of Tlr4-specific RNA prepared from AECs (Figure 1c) and total splenocytes (Figure 1d). Inactivation of Tlr4 was also confirmed by in vitro and in vivo functional studies. Bone marrow-derived dendritic cells from $T l r 4^{f l f l}$ Shh-Cre mice and their Cre-negative $T l r 4^{f l / f l}$ littermates produced comparable amounts of tumor necrosis factor- $\alpha$ after treatment with LPS, whereas bone marrow-derived dendritic cells from $T l r 4^{f l f l}$ Vav1-Cre mice failed to produce this cytokine following LPS stimulation, although they responded strongly to the a

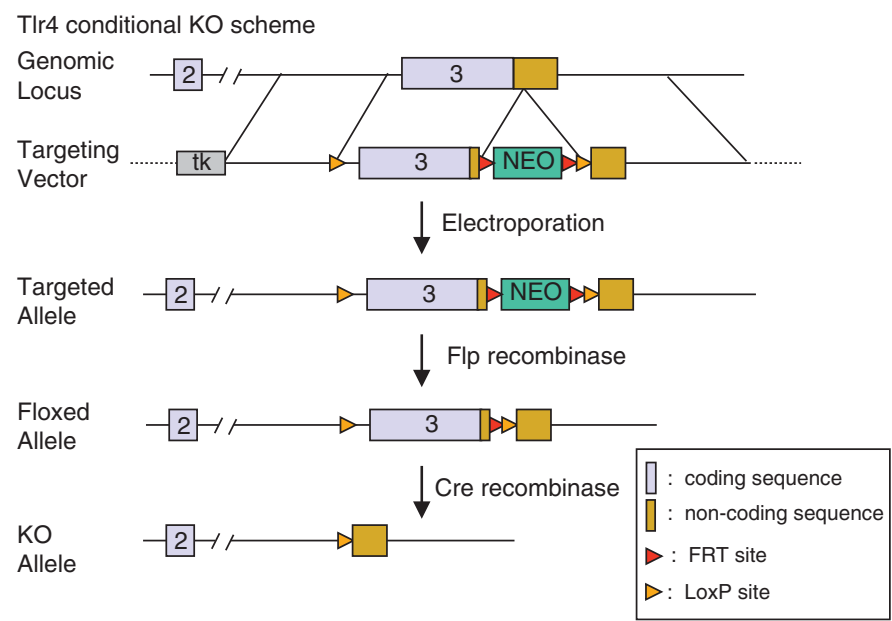

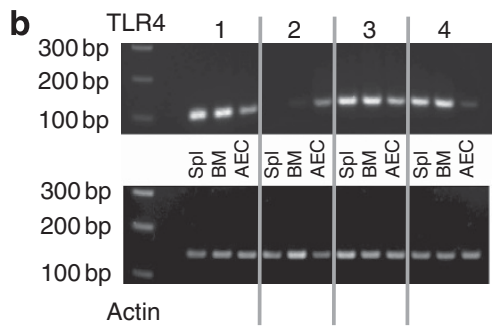

c
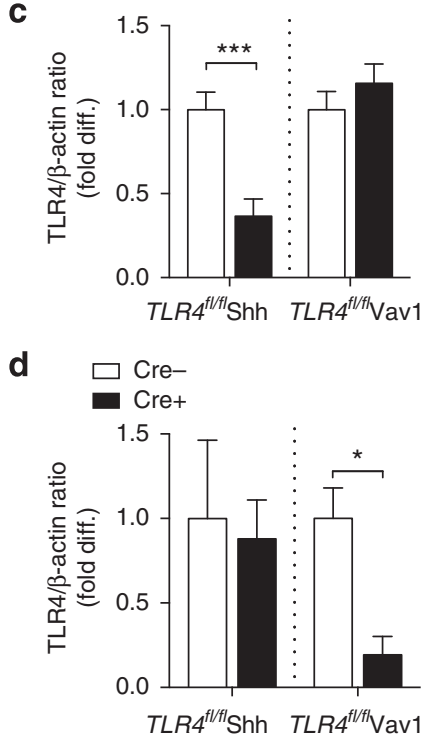

Figure 1 Generation of a T/r4 $4^{f / f l}$ mouse. (a) Structure of the targeting vector and strategy used to create TIr4 $4^{f / f l}$ mice. (b) PCR-based assay confirming selective deletion of TIr4 from genomic DNA from the indicated cell types. AEC, airway epithelial cell; BM, bone marrow; Spl, total splenocyte; TLR4, Toll-

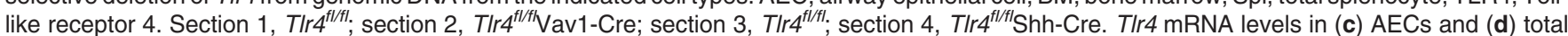
splenocytes. Data shown represent mean \pm s.e.m., and are from one experiment representative of two-three experiments. $N=4-6$ mice per group. ${ }^{\star} P<0.05 ;{ }^{* \star *} P<0.001$. 

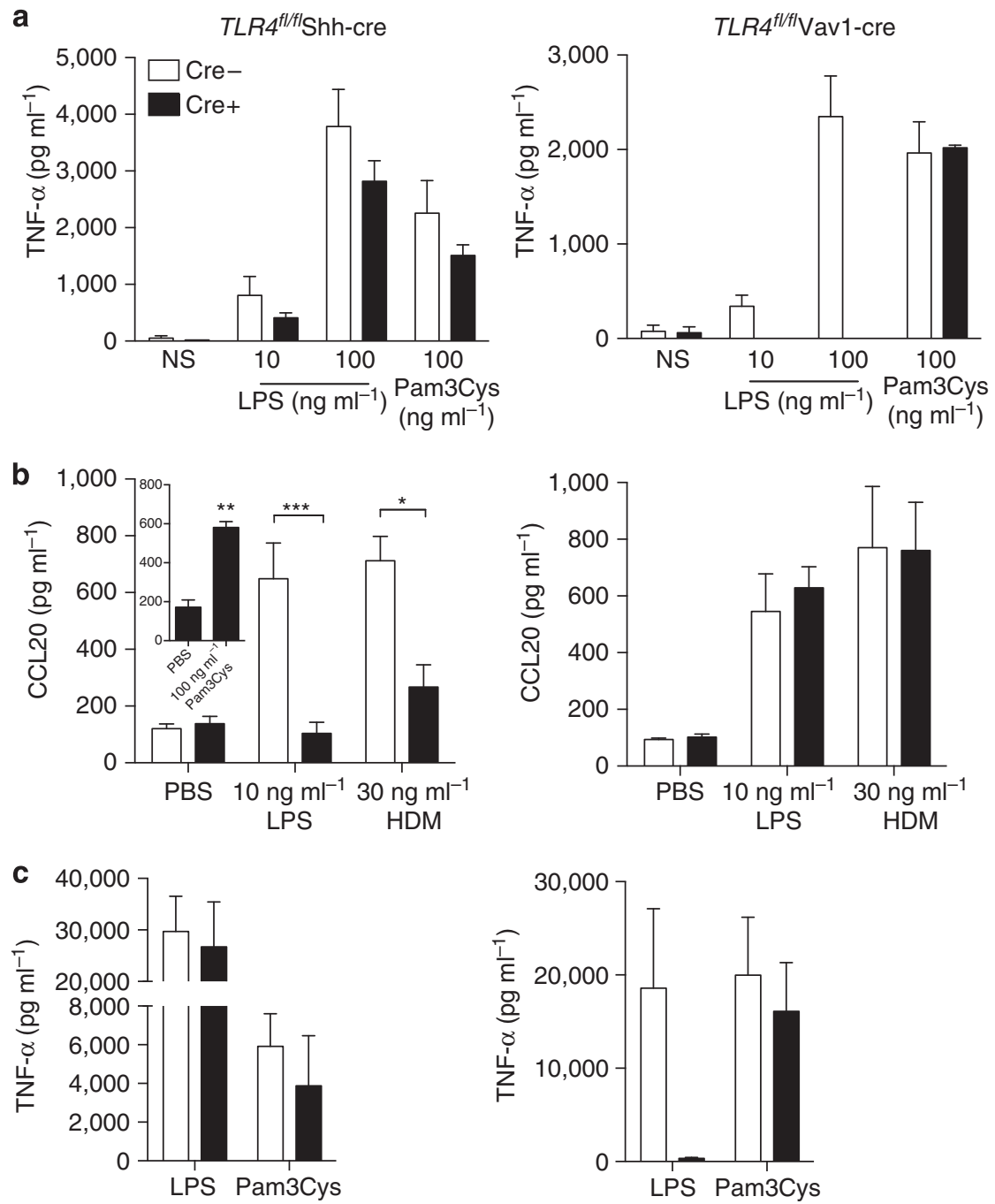

Figure 2 Lipopolysaccharide (LPS) responsiveness of cells from TLR4 $4^{f / f l}$ Shh-Cre and TLR4 $4^{f / f l}$ Vav1-Cre mice. (a) Tumor necrosis factor- $\alpha$ (TNF- $\alpha$ ) production by LPS- and Pam3Cys-treated bone marrow-derived dendritic cells (BMDCs; $n=4-6$ mice). (b) CCL20 production by LPS-, house dust mite (HDM)- and Pam3Cys-treated airway epithelial cells (AECs; $n=6-10$ mice/group). PBS, phosphate-buffered saline; TLR4, Toll-like receptor 4. (c) Serum TNF- $\alpha$ in mice $24 \mathrm{~h}$ after intraperitoneal (IP) injection of LPS ( $n=6-8$ mice/group). Data represent mean \pm s.e.m. from one of two-three experiments yielding similar results. ${ }^{\star} P<0.05 ;{ }^{\star \star} P<0.01 ;{ }^{* \star *} P<0.001$.

TLR2 ligand, Pam3Cys (Figure 2a). Conversely, AECs from $T l r 4^{f l f l}$ Vav1-Cre and Tlr $4^{f l f l}$ mice produced comparable levels of the epithelium-derived chemokine, CCL20, in response to LPS or house dust mite (HDM) challenge, whereas AECs from $T l r 4^{f l / f l} \mathrm{Shh}$-Cre mice had dramatically reduced responses to these stimuli (Figure 2b). Finally, Tlr $4^{f l / f l}$ Shh-Cre mice, but not $T l r 4^{f l f l}$ Vav1-Cre, exhibited high levels of serum tumor necrosis factor- $\alpha$ after intraperitoneal injection of LPS mice (Figure 2c). Taken together, these data indicate efficient functional deletion of TLR4 in AECs of Tlr $4^{f l / f l}$ Shh-Cre mice and in hematopoietic cells of Tlr $4^{f l / f l}$ Vav1-Cre mice.

\section{TIr4 expression by hematopoietic cells promotes LPS-mediated airway inflammation}

Previous studies employing bone marrow chimeric mice to address the relative contribution of different Tlr4-expressing cell populations to LPS responsiveness have led to diverse conclusions. ${ }^{16-18}$ To address this issue without the complications associated with irradiation, we administered ultrapure LPS to Tlr $4^{f l / f l}$ Shh-Cre mice and Tlr $4^{f l / f l}$ Vav1-Cre mice by intratracheal aspiration. Two peaks of neutrophil accumulation were seen in the airways of Tlr $4^{f l / f l}$ and $T l r 4^{f l / f l}$ Shh-Cre mice; at 4 and $24 \mathrm{~h}$ after LPS treatment (Figure 3a). However, at both of these time points, $\operatorname{Tlr} 4^{f l / f l}$ Vavl-Cre mice had significantly reduced numbers of these neutrophils, regardless of the amount of LPS used (Figure $\mathbf{3 b}$ ). Thus, in nonirradiated mice, hematopoietic cell-specific Tlr4 expression is critical to LPSmediated neutrophil recruitment to the airway and AECspecific expression of this gene is dispensable. Tlr $4^{f l / f} \mathrm{Shh}$ - and Vav1-Cre mice also had a trend toward reduced neutrophil recruitment to the lung following phosphate-buffered saline treatment when compared with phosphate-buffered 
a

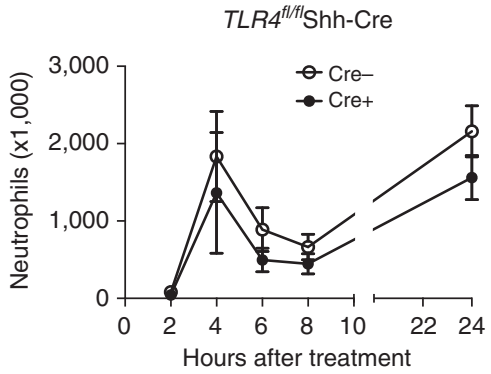

b

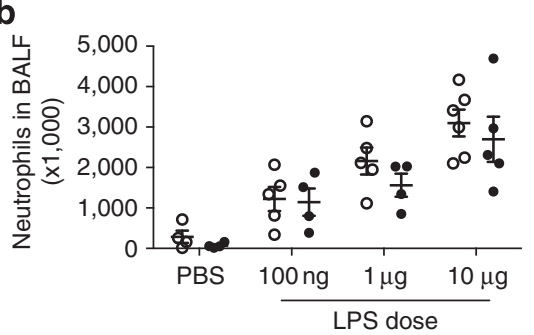

C

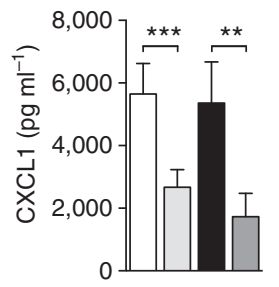

d

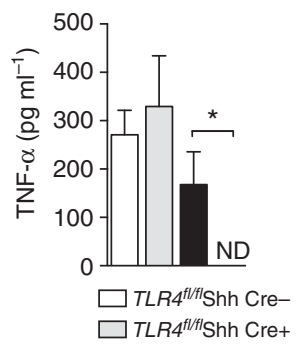

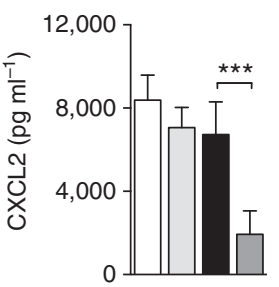

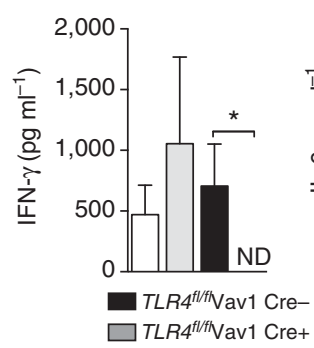

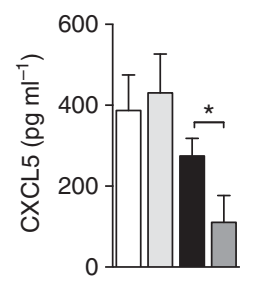

$T L R 4^{f / / f I}$ Vav1-Cre
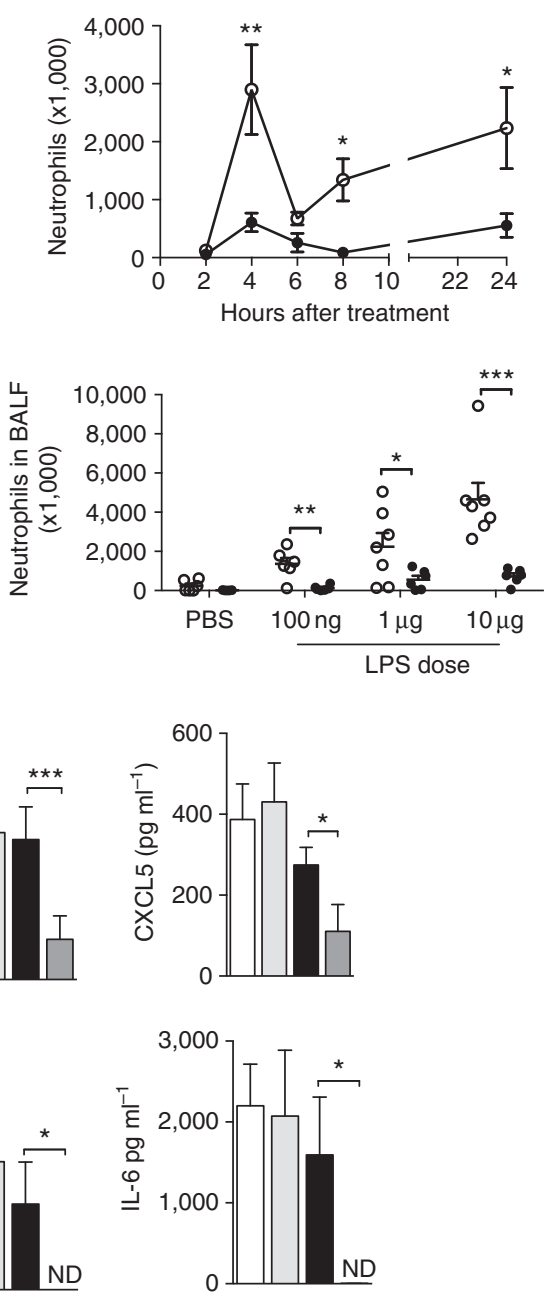

Figure 3 TIr4-expressing hematopoietic cells promote lipopolysaccharide (LPS)-mediated neutrophilic inflammation. (a) Time course of neutrophil recruitment following inhalation of $1 \mu \mathrm{g}$ LPS. (b) LPS dose response for neutrophils in mice harvested $24 \mathrm{~h}$ after treatment. Chemokine and cytokine levels in the bronchoalveolar lavage fluid (BALF) at (c) $2 \mathrm{~h}$ and (d) $24 \mathrm{~h}$ after treatment with $1 \mu \mathrm{g} \mathrm{LPS}$. IFN- $\gamma$, interferon- $\gamma$; IL-6, interleukin-6; ND, not detectable; PBS, phosphate-buffered saline; TLR4, Toll-like receptor 4; TNF- $\alpha$, tumor necrosis factor- $\alpha$. Data represent mean \pm s.e.m. from one experiment representative of three experiments, with $4-12$ mice per group. ${ }^{*} P<0.05 ;{ }^{* \star} P<0.01 ;{ }^{* \star *} P<0.001$.

saline-treated littermate controls, although these differences did not reach statistical significance. This trend might have been because of the role of Tlr4 in neutrophilic responses to minor injury caused by repeated fluid installation into the lungs. ${ }^{26}$

The markedly reduced neutrophil accumulation seen in $T l r 4^{f l f l}$ Vav1-Cre mice following LPS instillation suggested that neutrophil-attracting chemokines might also be reduced in these animals. To test this, we instilled LPS into the airways of mice and assayed bronchoalveolar lavage (BAL) fluid for CXCL1, CXCL2, and CXCL5. Each of these chemokines were significantly reduced in the airways of Tlr $4^{f l f l}$ Vav1-Cre mice compared with $T l r 4^{f l f l}$ littermates, whereas concentrations of these chemokines in $T l r 4^{f l / f l}$ Shh-Cre mice were generally similar to their nontransgenic littermates (Figure 3c). An exception was the reduction in CXCL1 seen in Tlr $4^{f l / f l} \mathrm{Shh}$-Cre mice, although this reduction was apparently insufficient to significantly affect the number of neutrophils recruited to the airways. In addition to the reduction in amounts of neutrophilattracting chemokines in the airway, Tlr $4^{f l / f l}$ Vav1-Cre mice also had significantly reduced amounts of interferon- $\gamma$, tumor necrosis factor- $\alpha$, and interleukin (IL)- 6 in BAL fluid $24 \mathrm{~h}$ after challenge (Figure 3d), cytokines whose levels were unaffected in Tlr $4^{f l f l}$ Shh-Cre mice. Collectively, these data demonstrate that hematopoietic cell expression of Tlr4 is critical for LPSdriven recruitment of neutrophils to the airway.

\section{TIr4 expression by hematopoietic cells regulates neutrophilic responses to HDM}

HDMs represent a ubiquitous source of household allergens to which many patients with allergic asthma are sensitized. To determine the roles of Tlr4-expressing AECs and hematopoietic 
cells in acute immune responses to HDM, we challenged the airways of mice with HDM and evaluated airway inflammation $24 \mathrm{~h}$ later. As observed after LPS treatment, HDM-treated $T l r 4^{f l / f l}$ Vav1-Cre mice had significantly fewer airway neutrophils than did their Tlr $4^{f l f l}$ littermate controls, whereas Tlr $4^{f l}$ ${ }^{f l}$ Shh-Cre mice and control Tlr $4^{f l f l}$ littermates had similar numbers of neutrophils (Figure 4a). To determine a possible cause for the decreased number of neutrophils recruited to the airways of Tlr $4^{f l / f l}$ Vav1-Cre mice, we quantified CXCL1 and CXCL2 in the BAL fluid. Both chemokines were reduced in $T l r 4^{f l f l}$ Vav1-Cre mice when compared with nontransgenic littermates (Figure $\mathbf{4 b}$ ), whereas these chemokines were similar in $\operatorname{Tlr} 4^{f l / f l}$ Shh-Cre mice and nontransgenic Tlr $4^{f l / f l}$ controls. Because IL-25 and IL-33 have been implicated in allergic sensitization through the airway, ${ }^{27-32}$ we also quantified these cytokines in HDM-treated mice. Tlr $4^{f l / f}$ Shh-Cre mice had reduced levels of IL-25 and IL-33 (Figure 4c) in the BAL fluid, compared with $T l r 4^{f l f l}$ littermate controls, whereas Tlr $4^{f l /}$ ${ }^{f l}$ Vav1-Cre mice and their Tlr $4^{f l f l}$ littermates had similar amounts of these cytokines. Thus, Tlr4-expressing hematopoietic cells produce neutrophil-attracting chemokines in response to LPS, whereas Tlr4-expressing AECs produce Th2-promoting cytokines.
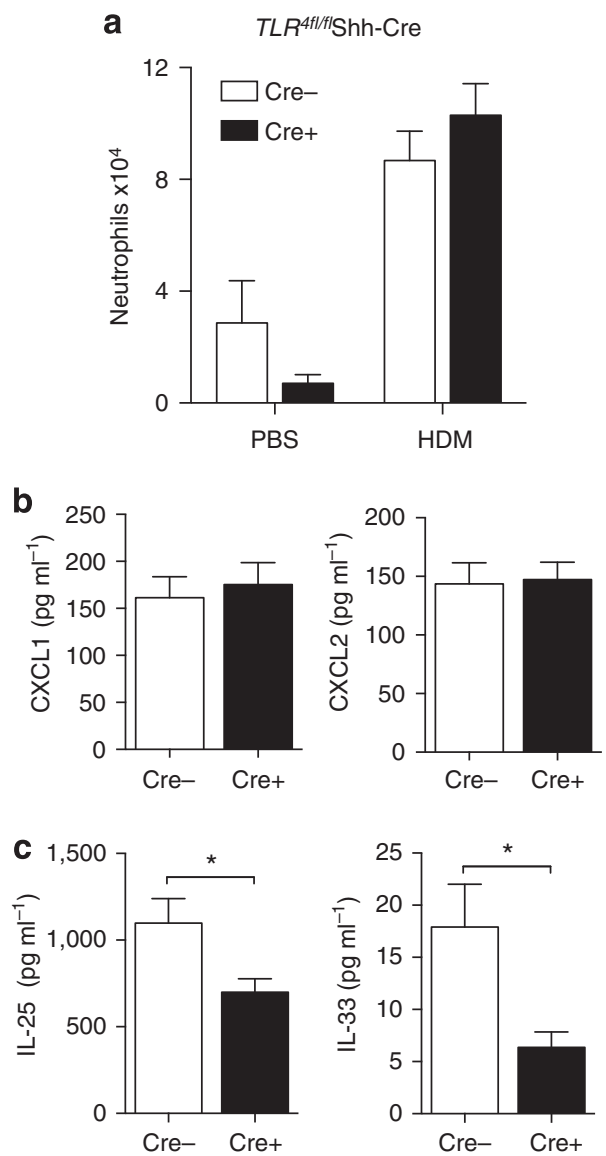

TLR4-expressing airway epithelial and hematopoietic cells differentially regulate eosinophilic and neutrophilic allergic airway inflammation

To determine the role of TLR4 expression by airway epithelial and hematopoietic cells in a clinically relevant mouse model of asthma, we sensitized Tlr $\Psi^{f l / f l}$ Shh-Cre mice and Tlr $4^{f l /}$ ${ }^{f l}$ Vav1-Cre mice with HDM on days 0 and 7, followed by challenge with this same allergen preparation on day 14 . The commercial preparation of HDM we used contains $\sim 100 \mathrm{ng}$ LPS per $100 \mu \mathrm{g}$ of HDM, sufficient to promote adaptive immune responses to bystander allergens and drive Tlr4dependent eosinophilia and neutrophilia in several mouse strains $^{13,14,18,33}$ (Figure 5a). No differences between either of the present strains and their Tlr $4^{f / f l}$ littermates were seen when total leukocytes in the airway were quantified (Figure $\mathbf{5 b}, \mathbf{c}$ ). However, cell differential analysis of the leukocytes in BAL revealed marked differences among these strains. Tlr $4^{f l f l}$ Vav1-Cre mice had significantly fewer neutrophils in their airways than did their Tlr $4^{f l / f l}$ littermates, whereas Tlr $4^{f l / f l}$ Shh-Cre mice had similar numbers of neutrophils compared with their nontransgenic littermates. Conversely, eosinophil numbers were significantly reduced in $T l r 4^{f / f l}$ Shh-Cre mice compared with their Tlr $\Psi^{\text {fl/fl }}$ littermates,
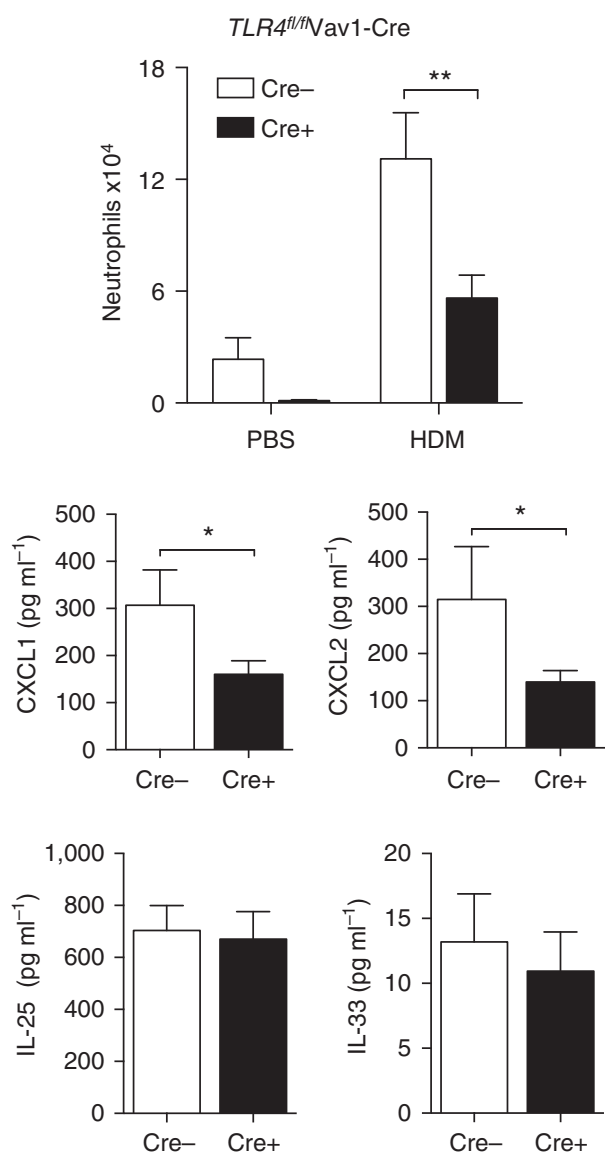

Figure 4 Role of TIr4-expressing airway epithelial cells (AECs) and hematopoietic cells in innate immune responses to house dust mite (HDM). (a) Neutrophils in bronchoalveolar lavage (BAL) fluid of mice $24 \mathrm{~h}$ after a single instillation of HDM. (b) CXCL1 and CXCL2 and (c) interleukin (IL)-25 and IL-33 levels in the BAL fluid. PBS, phosphate-buffered saline; TLR4, Toll-like receptor 4. Data represent mean \pm s.e.m. from one experiment representative of two experiments, with 6-8 mice per group. ${ }^{*} P<0.05 ;{ }^{*} P<0.01$. 

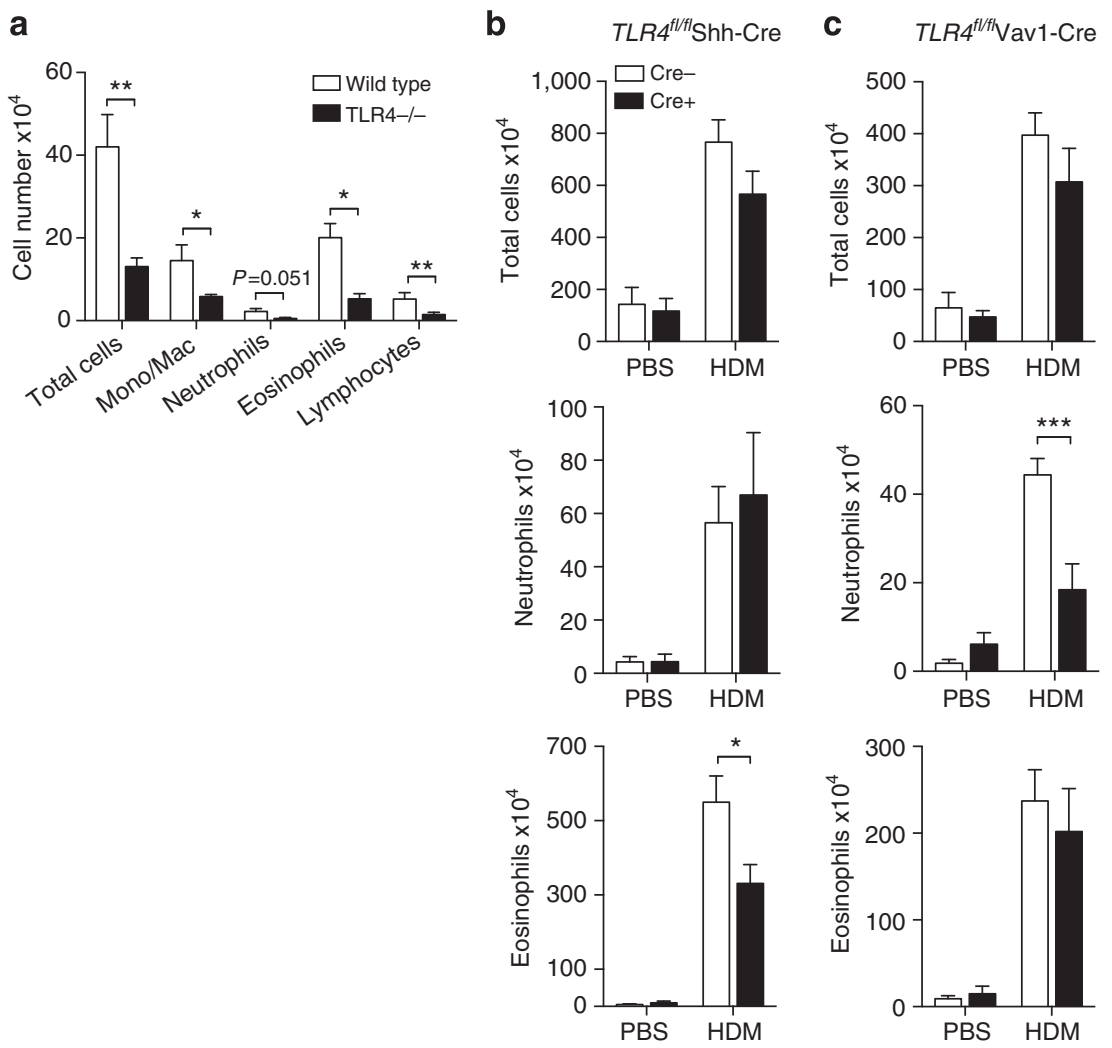

Figure 5 TIr4-expressing airway epithelial cells (AECs) and hematopoietic cells control distinct aspects of house dust mite (HDM)-driven allergic airway inflammation. Airway inflammation in the HDM model of asthma. (a) Mean cell numbers \pm s.e.m. are shown for the indicated cell types in bronchoalveolar lavage (BAL) fluid from wild-type (WT) and TIr4 ${ }^{-1-}$ mice. Data are from one experiment representative of two experiments.

(b) $T / r 4^{f / / f l} S h h-C r e$ and (c) T/r4 ${ }^{\mathrm{fl} / \mathrm{l}}$ Vav1-Cre mice sensitized and challenged with HDM extracts. PBS, phosphate-buffered saline; TLR4, Toll-like receptor 4. Values for the indicated cell types represent mean cell numbers \pm s.e.m. from one experiment representative of three experiments. $N=6-12$ mice/ group. ${ }^{*} P<0.05 ;{ }^{* \star} P<0.01 ;{ }^{* \star *} P<0.001$.

whereas the numbers of these cells were similar in Tlr $4^{f l / f l}$ Vav1Cre and Tlr $4^{f l / f l}$ mice. Thus, Tlr4 expression in specific cell compartments affects distinct arms of the airway immune response, with expression in hematopoietic cells promoting neutrophilic inflammation and expression in epithelial cells promoting eosinophilic inflammation.

To examine whether differences in cytokine and chemokine levels might have led to qualitative differences in airway inflammation, whole lungs from HDM-sensitized and -challenged mice were restimulated in vitro with HDM. Surprisingly, IL-33 was the only cytokine that we found significantly reduced in the lungs of Tlr $\Psi^{f l f l}$ Shh-Cre mice compared with Tlr $4^{f l / f l}$ littermate controls (Figure 6a). Lungs from Tlr $4^{f l / f l}$ Vav1-Cre mice also had reduced amounts of IL-33 (Figure $6 \mathbf{b}$ ), and produced significantly less of the neutrophilia-promoting cytokines, IL-17A and IL-17F.

To compare our study of Tlr $4^{f l / f l}$ mice with previous studies using bone marrow chimeric mice, we used wild-type (WT) and $\mathrm{Tlr} 4^{-1-}$ animals to generate reciprocal bone marrow chimeras and studied them in the HDM-driven model of allergic asthma. As expected, when WT marrow was transferred into irradiated WT recipients $(\mathrm{WT} \rightarrow \mathrm{WT})$, HDM-treated mice developed both eosinophilic and neutrophilic airway inflammation (Figure 7a). However, WT $\rightarrow T l r 4^{-1-}$ mice displayed very little eosinophilia, in agreement with our finding of reduced eosinophilia in Tlr $\Psi^{f l / f l}$ Shh-Cre mice and confirming an important role of Tlr4 expression in airway structural cells for eosinophilic inflammation. Conversely, neutrophilic airway inflammation was not significantly affected in the WT $\rightarrow$ $\mathrm{Tlr} 4^{-1-}$ mice, but was completely abrogated in $\mathrm{Tlr} 4^{-1-} \rightarrow$ WT mice, whereas eosinophils were unaffected. This finding was also consistent with our observation that neutrophilia is reduced in Tlr $4^{f l f l}$ Vav1-Cre mice, confirming that Tlr4 expression in hematopoietic cells is critical for the neutrophilic component of airway inflammation. Analysis of cytokines in lungs prepared from bone marrow chimeric mice revealed that, as seen in the Tlr $4^{f l f l}$ model, Tlr4 expression in hematopoietic cells is critical for production of IL-17A and IL-17F (Figure 7b).

To confirm that our results were not unique to the HDM model of allergic inflammation, we carried out experiments in a different established mouse model of asthma in which inhaled LPS promotes Th2 and Th17 responses to inhaled OVA. These experiments confirmed that, as we had seen with the HDM model, Tlr4 expression in hematopoietic cells is critical for production of IL-17A and IL-17F and associated neutrophilic 
inflammation, whereas Tlr4 expression in hematopoietic cells is critical for production of IL-5 and eosinophilic inflammation (Figure 8).
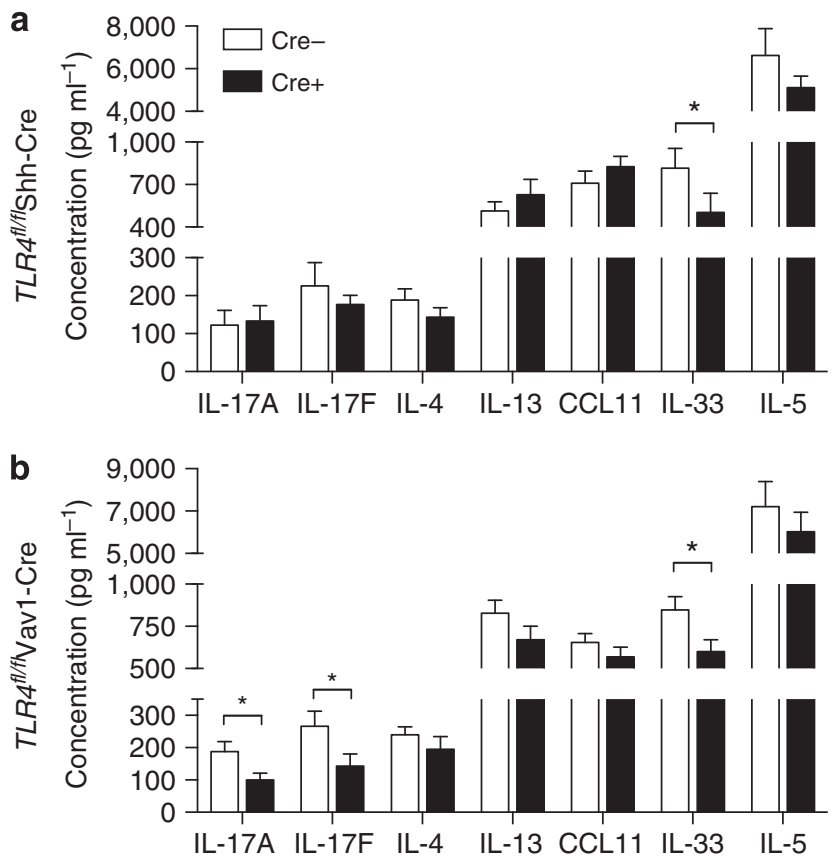

Figure 6 Effect of TIr4-expressing cell types on cytokine and chemokine production in the lung. Cytokines and chemokines in the supernatants of whole lungs restimulated ex vivo for 5 days with house dust mite (HDM) from (a) TIr $4^{\mathrm{fl} / \mathrm{fl}} \mathrm{Shh}$-Cre and (b) $T / \mathrm{r} 4^{\mathrm{fl} / \mathrm{fl}}$ Vav1-Cre mice. IL, interleukin; TLR4, Toll-like receptor 4. Data represent mean concentrations \pm s.e.m. from one experiment representative of two experiments. $N=6-12$ mice/ group. ${ }^{\star} P<0.05$.

\section{DISCUSSION}

Previous studies employing bone marrow chimeric mice have led to diverse conclusions regarding the contribution of Tlr4expressing cell compartments to leukocyte recruitment in animal models of acute inflammation and allergic airway inflammation. Some of this diversity is likely because of variable effects of radiation on structural and hematopoietic cell function. ${ }^{20}$ In this study, we circumvented this complication by generating Tlr $4^{f l / f l}$ mice in which deletion of Tlr4 signaling can be achieved in specific cell types without irradiation. With regard to acute immune responses to inhaled LPS, Tlr $4^{f l f l}$ Vav1-Cre mice displayed significant reductions in airway neutrophilia (and the neutrophil chemokines CXCL1, CXCL2, and CXCL5), indicating that Tlr4 expression by hematopoietic cells is important for airway neutrophilic immune responses. However, neutrophilic inflammation was not completely absent in $T l r 4^{f l}$ ${ }^{f l}$ Vav1-Cre mice, and Tlr $4^{f l / f l}$ Shh-Cre mice lacking functional Tlr4 in airway AECs had modest reductions in neutrophilia and CXCL1. Thus, in these experiments, Tlr4 expression by hematopoietic cells was primarily responsible for neutrophil recruitment to airways in both genetic and radiation chimera models of hematopoietic cell deletion of TLR4 expression. This conclusion is concordant with a previous study of bone marrow chimeric mice by Hollingsworth et al., ${ }^{16}$ but in opposition to the results of a bone marrow chimera study by Hammad et al. ${ }^{17}$ that reported airway structural cells were necessary and sufficient for airway neutrophilia. The reasons underlying the latter, discrepant, results remain unclear.

A novel finding of our study is that in mouse models of allergic asthma, Tlr4 expression by hematopoietic cells and AECs drives distinct arms of the airway immune responses.
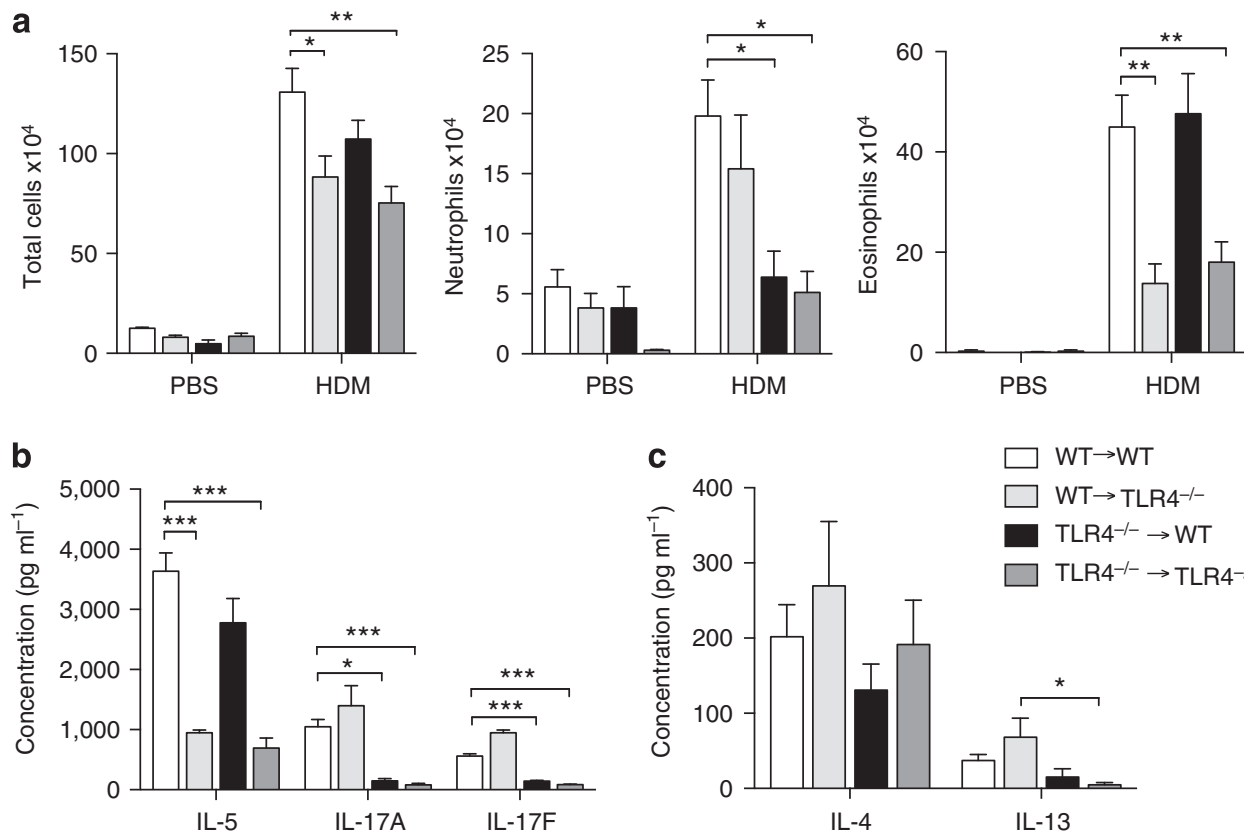

Figure 7 Allergic responses to house dust mite (HDM) sensitization and challenge in bone marrow chimeric mice. (a) Airway inflammation and (b, c) cytokine production in the HDM model of asthma. Cytokines are from cultures of whole lungs restimulated ex vivo for 5 days with HDM. IL, interleukin; PBS, phosphate-buffered saline; TLR4, Toll-like receptor 4; WT, wild type. Data represent mean \pm s.e.m. from one experiment representative of three experiments. ${ }^{\star} P<0.05 ;{ }^{*} P<0.01 ;{ }^{* *} P<0.001$. 

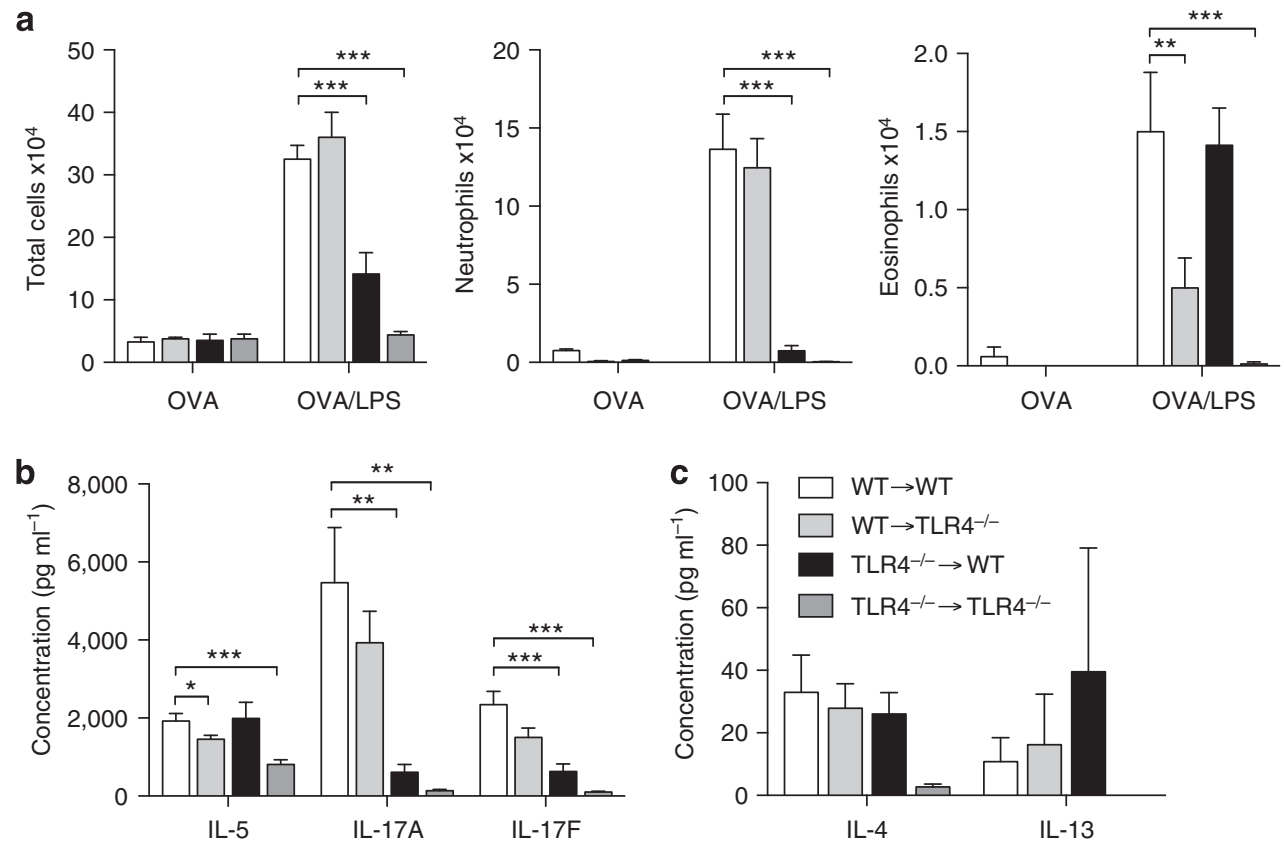

Figure 8 Allergic responses to ovalbumin/lipopolysaccharide (OVA/LPS) sensitization and challenge in bone marrow chimeric mice. (a) Airway inflammation and (b, $\mathbf{c})$ cytokine production in the OVA/LPS model of asthma. Cytokines are from cultures of whole lungs restimulated ex vivo for 5 days with OVA. IL, interleukin; TLR4, Toll-like receptor 4; WT, wild type. Data shown represent mean \pm s.e.m., and are from one experiment representative of three experiments. $N=2-4$ mice/per group. ${ }^{\star} P<0.05 ;{ }^{\star \star} P<0.01 ;{ }^{* \star} P<0.001$.

Specifically, whereas neutrophil recruitment following allergen sensitization and challenge was primarily dependent on hematopoietic cell expression of Tlr4, eosinophilic inflammation was chiefly dependent on Tlr4 expression by AECs. Moreover, this dichotomy was observed in two different models of allergic asthma, suggesting that TLR4 signaling in these two cell types leads to fundamentally distinct outcomes. The deficit in neutrophil recruitment after HDM sensitization and challenge was more pronounced in Tlr4 ${ }^{-1-} \rightarrow \mathrm{WT}$ bone marrow chimeras than in Tlr $4^{f l f l}$ Vav1-Cre mice, possibly because structural cells other than AECs are damaged by radiation and therefore less responsive to LPS in chimeric mice. The decreased airway neutrophilia observed in mice lacking TLR4 in hematopoietic cells was associated with decreased concentrations of IL-17A and IL-17F, cytokines that are known to promote neutrophil accumulation in the lungs. ${ }^{14,34,35}$ This finding is consistent with a previous report showing that with high doses of LPS (10-15 $\mu \mathrm{g})$, Tlr 4 expression by hematopoietic cells is primarily responsible for the production of IL-17 in an OVA/LPS model of asthma. ${ }^{18}$ A similar effect was not seen when lower dose of $100 \mathrm{ng}$ LPS was used, possibly because the timing of the OVA/LPS sensitizations used in that study leads to a weaker Th17-mediated neutrophilic response to OVA challenge than does the protocol employed in our study (G.S. Whitehead et al, unpublished observations). IL-17A and IL-17F induce neutrophil recruitment to the airways by inducing chemokine production, primarily by AECs. ${ }^{36}$ Taken together with these previous observations, our current findings suggest that chemokine production by AECs is at least partially dependent on TLR4 responsiveness in hematopoietic cells. The concept of cross-talk between AECs and hematopoietic cells has been proposed previously, ${ }^{37,38}$ but there is only limited understanding of the specific pathways that might be involved. The conditionally targeted Tlr $4^{f l f l}$ mice provide a novel resource to study how functional deletion of Tlr4 in one cell type can alter the function of other cell types.

Studies of $T l r 4^{f l f l}$ mice and bone marrow chimeric mice support previous findings that Tlr4 expression by AECs strongly contributes to eosinophilic component of allergendriven airway eosinophilia. This finding is consistent with previous work with bone marrow chimeric mice in the $\mathrm{HDM}^{17}$ and OVA/LPS ${ }^{18}$ models of allergic asthma. However, the reduction in eosinophils was less marked in the Tlr $\Psi^{f l / f l} \mathrm{Shh}$-Cre mice than in the WT $\rightarrow$ Tlr $4^{-1-}$ chimeric mice, suggesting that structural cells other than AECs also contribute to HDM-mediated eosinophilic response. Our present study also confirms previous results suggesting that the absence of Tlr4 expression in hematopoietic cells does not significantly affect HDM-driven airway eosinophilia. ${ }^{17}$

A previous study attributed decreased eosinophilia in $\mathrm{WT} \rightarrow$ $\mathrm{Tlr}^{-1-}$ bone marrow chimeric mice to reduced $\mathrm{MHCII}^{+}$ $\mathrm{CD}_{11 \mathrm{~b}^{+}}$dendritic cell activation and reduced granulocyte colony-stimulating factor, thymic stromal lymphopoietin, IL25 , and IL-33 production after HDM sensitization. Here, we also found that selective deletion of functional Tlr4 from AECs led to reduced IL-25 and IL-33 in the BAL fluid $24 \mathrm{~h}$ after a single HDM instillation. Thus, reductions in these cytokines might have contributed to decreased eosinophilia seen after 
allergen challenge because they are reported to promote development of Th2-type immune responses that drive eosinophilia in the lung. ${ }^{39,40}$ However, the amounts of IL-4, IL-13, and IL-5 were comparable in lungs of HDM-challenged Tlr $4^{\text {fl/fl }}$ Shh-Cre mice and their nontransgenic TLR4 $4^{f l f l}$ littermates, suggesting that Th2 cell development might not be affected by the lack of Tlr4 expression in AECs. Unlike in Tlr $4^{f l / f l}$ Shh-Cre mice, IL-5 was significantly reduced in lungs of HDMsensitized and -challenged WT $\rightarrow T l r 4^{-1-}$ bone marrow chimeric mice compared with WT $\rightarrow \mathrm{WT}$ control animals, whereas IL- 4 and IL-13 were not significantly affected. This suggests that Tlr4-expressing radioresistant cells in the lungother than AECs-regulate IL-5 production. These data extend previous studies by showing that TLR4 expression in AECs is necessary for allergen-driven lung eosinophilia, although the molecular mechanisms that drive this response in the present model remain unclear.

Our novel finding that Tlr4 expression in hematopoietic cells and AECs direct distinct arms of the airway immune response has important implications for the therapeutic treatment of individuals with distinct forms of asthma. Global blockade of TLR4 signaling in the airway has shown promise for diminishing allergic sensitization, ${ }^{17}$ but this approach might impair the function of cells that are important for host defense against pathogens. Selectively targeting TLR4 signaling in specific cell types that promote either neutrophilic or eosinophilic forms of asthma might reduce the severity of this disease while minimizing effects on host defense. Such a targeted approach might be particularly important for neutrophilic forms of asthma that are resistant to glucocorticoid therapy. ${ }^{4-6}$ We have not yet identified the specific hematopoietic cell type(s) expressing Tlr4 that are necessary for the induction of neutrophilic inflammation. Dendritic cells represent a compelling hypothesis in this regard-responding to LPS by increasing costimulatory molecule expression and the production of cytokines such as IL- 1 and IL- 6 that promote the development of allergen-specific Th17 cells, in turn promoting neutrophilic asthma. However, macrophages, innate lymphoid, and/or $\alpha / \beta$ $\mathrm{T}$ cells might also play a role. Defining roles for Tlr4 expression in each of these cell types awaits further generation of appropriate cell-specific Cre TLR4-floxed crosses. Identification of the specific hematopoietic cell type(s) requiring Tlr4 expression for the induction of neutrophilic asthma will be critical for a deeper understanding of asthma and for designing novel strategies for therapeutic intervention.

\section{METHODS}

Mice. Animals were housed under specific pathogen-free conditions at the National Institute of Environmental Health Science or Cincinnati Children's Research Foundation and used between 5 and 12 weeks of age. All animal experiments were conducted in accordance with the Institutional Animal Care and Use Committee of the respective institutions.

Mice expressing Cre recombinase in the Shh locus (B6.Cg$S h h^{\mathrm{tml} 1 \text { (EGFP/cre)Cjt } / J)}$ or from the Vav1 promoter B6.Cg-Tg(Vav1-Cre) A2Kio/J, as well as C57BL/6J, Tlr4 ${ }^{-1-}$ (B6.B10ScN-Tlr $4^{\text {lps-del } / J t h J), ~}$ $\mathrm{B} 6(\mathrm{Cg})-\mathrm{Tyr}^{c-2 J} / \mathrm{J}$, and B6.Cg-Tg(ACTFLPe)9205Dym/J mice, were purchased from Jackson Laboratories (Bar Harbor, ME). The Creexpressing mice were bred to conditionally mutant, Tlr $4^{f l / f l}$ mice generated in collaboration with the Gene Targeted Mouse Service Core at the University of Cincinnati and are available at Jackson Laboratories (strain \#024872). The Tlr4 targeting vector included two LoxP sites flanking exon 3, an FRT-flanked neomycin resistance gene $(\mathrm{Neo})$ for positive selection, a thymidine kinase gene for negative selection, and 1.9 and $3.4 \mathrm{~kb}$ arms of genome-derived DNA homologous with the Tlr4 locus. The targeting vector was introduced by electroporation into C57BL/6 mouse embryonic stem cells, and drug-resistant clones were screened by a PCR assay specific for the targeted Tlr4 gene. Two cell lines whose correctly targeted Tlr $4^{f l}$ locus had been confirmed by Southern blot analysis were used to generate chimeras by injection into blastocysts from albino C57BL/6 (B6 $(\mathrm{Cg})$ $\left.\mathrm{Tyr}^{\mathrm{c}-2 J} / \mathrm{J}\right)$ mice. Two such chimeras were chosen to breed with B6(Cg)$\mathrm{Tyr}^{\mathrm{c}-2 J} / \mathrm{J}$ mice, and embryonic stem cell-derived offspring were identified by their black coat color. Black mice bearing the targeted Tlr4 gene were then bred to FlpE recombinase-encoding B6.Cg$\mathrm{Tg}$ (ACTFLPe)9205Dym/J mice to delete neo from the Tlr4 locus. Offspring with the target Tlr4 locus, but lacking neo, were crossed to

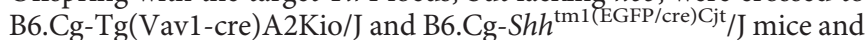
the offspring interbred to obtain homozygous $T l r 4^{f l f l}$ mice with the desired Cre-expressing transgene.

Reciprocal bone marrow chimeric animals were generated using WT C57BL/6J and Tlr4 ${ }^{-1-}$ donors and recipient mice. Recipient animals were irradiated at 6-8 weeks of age with 900 rad over $12 \mathrm{~min}$ and injected 1 day later with $10^{7}$ donor bone marrow cells. Mice were given acidified water, supplemented with $500 \mathrm{\mu g} \mathrm{ml}^{-1}$ neomycin for 2 weeks after irradiation. The animals were used in experiments after allowing for a minimum of 10 weeks for hematopoietic reconstitution, as confirmed by flow cytometry (data not shown).

Models of airway disease. Mice were anesthetized with inhaled isoflurane and all reagents were administered via intratracheal aspiration. ${ }^{41}$ To measure innate immune responses, mice were administered a single dose of $100 \mu \mathrm{g}$ of Dermatophagoides pteronyssinus extracts (Greer Laboratories, Lenoir, NC) or $1 \mu \mathrm{g}$ of TLR4-specific ultrapure LPS (Invivogen, San Diego, CA) in a total volume of $40 \mu \mathrm{l}$, and BAL was performed at 2, 4, 6, 8, or $24 \mathrm{~h}$ after challenge. For the model of HDM-mediated asthma, mice were given $100 \mu \mathrm{g}$ Dermatophagoides pteronyssinus extracts on days 0,7 , and 14 , and BAL performed on day 17. For the OVA/LPS model of asthma, mice were given $100 \mu \mathrm{g}$ LPS-depleted OVA (BioVendor. Candler, NC) together with $100 \mathrm{ng}$ LPS from Escherichia coli (Sigma, St Louis, MO) on days 0, 7 , and 14 and BAL was performed on day 16. Cell differential analysis was carried out as described previously. ${ }^{41}$ Lungs were cultured for $24 \mathrm{~h}$ in cRPMI ( $10 \%$ fetal bovine serum, $0.1 \%$ 2-mercaptoethanol, $1,000 \mathrm{Ul}^{-1}$ penicillin/streptomycin, $10-30 \mu \mathrm{g} \mathrm{ml}^{-1} \mathrm{HDM}$, or $10 \mu \mathrm{g} \mathrm{ml}^{-1} \mathrm{OVA}$ ), and cytokines in lung culture supernatants and BAL fluid (BALF) were quantified using Luminex or enzyme-linked immunosorbent assay (ELISA).

In vivo cytokine capture assay. Systemic cytokines were quantified using the in vivo cytokine capture assay as previously described. ${ }^{42}$ All antibodies and standards were from eBioscience (San Diego, CA).

AEC cultures. AECs were isolated and cultured as previously described. ${ }^{43}$ Briefly, 5-week-old tracheas were excised and disaggregated by $0.1 \%$ pronase (Roche, Indianapolis, IN) and DNase I (Sigma) digestion, followed by fibroblast removal by plastic adherence. Mouse tracheal epithelial cells (MTECs) were submersion cultured on type I collagen-coated, $0.4 \mu \mathrm{m}$ pore transwell inserts (Corning, Tewksbury, MA) until formation of tight junctions ( $\mathrm{R} \geq 1,000 \Omega$; 7-14 days). An air-liquid interphase was established and maintained with daily basolateral media changes for 7 days before the AECs were stimulated apically with $10-100 \mathrm{ng} \mathrm{ml}^{-1}$ TLR4-specific ultrapure E. coli K12 LPS, $100 \mathrm{ng} \mathrm{ml}^{-1}$ Pam3CSK4 (Invivogen), $30 \mathrm{mg} \mathrm{ml}^{-1}$ HDM (Greer Laboratories), or vehicle control. Basolateral chemokine 
and cytokine production was quantified by commercially available Luminex or ELISA assays.

Bone marrow-derived dendritic cell culture. Dendritic cells were generated from bone marrow using standard protocols as previously described. ${ }^{44}$ Cells were stimulated with LPS or Pam3Cys at the indicated concentrations for $24 \mathrm{~h}$ and cytokine levels in supernatants were quantified by commercially available ELISA.

Cytokines. Cytokine concentrations in sample supernatants were quantified by ELISA using commercially available ELISA kits from R\&D Systems (Minneapolis, MN) and BD Biosciences (San Jose, CA) or using Milliplex Multiplex kits (Millipore, Billerica, MA) according to the manufacturer's protocol and read using luminex technology on the Bio-Plex (Bio-Rad, Hercules, CA). Concentrations were calculated from standard curves using recombinant proteins and expressed in $\operatorname{pg}(\mathrm{ng}) \mathrm{ml}^{-1}$.

Gene expression analysis by quantitative PCR. RNA was extracted using TRIzol Reagent (Invitrogen, Waltham, MA) according to the manufacturer's instructions. Complementary DNA was generated as previously described ${ }^{45}$ and subjected to quantitative PCR analysis using Light Cycler 480 II (Roche). Sybr Green I Master mix (Roche) and the following primers pairs were used: TLR4: $5^{\prime}$-CATCCAGG AAGGCTTCCACA- $3^{\prime}$ and $5^{\prime}$-GGCGATACAATTCCACCTGC-3'; $\beta$-actin: $5^{\prime}$-GCCCCAGAGCAAGAGAGGTA- $3^{\prime}$ and $5^{\prime}$-GGTTGGC CTTAGGTTTCAGG-3'.

Genomic DNA analysis. DNA was isolated from cells and tissues using the QIAamp DNA Mini Kit (Qiagen, Valencia, CA) according to the manufacturer's instructions and analyzed by PCR using the following primers: TLR4: 5'-CATCCAGGAAGGCTTCCACA- $3^{\prime}$ and $5^{\prime}$-GG CGATACAATTCCACCTGC- $3^{\prime}$; $\beta$-actin: $5^{\prime}$-CGCTCAGGAGGAGC AATGAT- $3^{\prime}$ and $5^{\prime}$-TCCTTAGCTTGGTGAGGGTG- $3^{\prime}$. Products were run on a $2 \%$ agarose gel and imaged.

Statistics. Data were analyzed by one-way analysis of variance, followed by Tukey's correction for multiple comparisons, or by unpaired Student's $t$-test, as appropriate.

\section{ACKNOWLEDGMENTS}

We thank Matt Edin and Michael Fessler (NIEHS) for critical reading of the manuscript and Traci Stankiewicz and Elizabeth Donelan (CCHMC) for technical assistance. This work was supported in part by the Intramural Research Program of the National Institutes of Health (NIH) and the NIEHS (to D.N.C.), NIH R01 Al088372 and R01 HL094576 (to C.L.K.), and F32 HL110497 (to J.W.M.)

\section{DISCLOSURE}

The authors declared no conflict of interest.

(c) 2015 Society for Mucosal Immunology

\section{REFERENCES}

1. McGrath, K.W. et al. A large subgroup of mild-to-moderate asthma is persistently noneosinophilic. Am. J. Respir. Crit. Care Med. 185, 612-619 (2012).

2. Barczyk, A., Pierzchala, W. \& Sozanska, E. Interleukin-17 in sputum correlates with airway hyperresponsiveness to methacholine. Respir. Med. 97, 726-733 (2003)

3. Bullens, D.M. et al. IL-17 mRNA in sputum of asthmatic patients: linking $\mathrm{T}$ cell driven inflammation and granulocytic influx? Respir. Res. 7, 135 (2006).

4. McKinley, L. et al. TH17 cells mediate steroid-resistant airway inflammation and airway hyperresponsiveness in mice. J. Immunol. 181, 4089-4097 (2008).

5. Green, R.H., Brightling, C.E., Woltmann, G., Parker, D., Wardlaw, A.J. \& Pavord, I.D. Analysis of induced sputum in adults with asthma: identification of subgroup with isolated sputum neutrophilia and poor response to inhaled corticosteroids. Thorax 57, 875-879 (2002).

6. Haldar, P. \& Pavord, I.D. Noneosinophilic asthma: a distinct clinical and pathologic phenotype. J. Allergy Clin. Immunol. 119, 1043-1052 (2007).

7. Lajoie, S. et al. Complement-mediated regulation of the IL-17A axis is a central genetic determinant of the severity of experimental allergic asthma. Nat. Immunol. 11, 928-935 (2010).

8. Karp, C.L. Guilt by intimate association: what makes an allergen an allergen?. J. Allergy Clin. Immunol. 125, 955-960 (2010).

9. Hoshino, K. et al. Cutting edge: Toll-like receptor 4 (TLR4)-deficient mice are hyporesponsive to lipopolysaccharide: evidence for TLR4 as the Lps gene product. J. Immunol. 162, 3749-3752 (1999).

10. Ross, M.A. et al. Association of asthma symptoms and severity with indoor bioaerosols. Allergy 55, 705-711 (2000).

11. Thorne, P.S., Kulhankova, K., Yin, M., Cohn, R., Arbes, S.J. Jr. \& Zeldin, D.C. Endotoxin exposure is a risk factor for asthma: the national survey of endotoxin in United States housing. Am. J. Respir. Crit. Care Med. 172, 1371-1377 (2005)

12. Braun-Fahrlander, C. et al. Environmental exposure to endotoxin and its relation to asthma in school-age children. N. Engl. J. Med. 347, 869-877 (2002).

13. Eisenbarth, S.C., Piggott, D.A., Huleatt, J.W., Visintin, I., Herrick, C.A. \& Bottomly, K. Lipopolysaccharide-enhanced, toll-like receptor 4-dependent T helper cell type 2 responses to inhaled antigen. J. Exp. Med. 196, 1645-1651 (2002).

14. Wilson, R.H., Whitehead, G.S., Nakano, H., Free, M.E., Kolls, J.K. \& Cook D.N. Allergic sensitization through the airway primes Th17-dependent neutrophilia and airway hyperresponsiveness. Am. J. Respir. Crit. Care Med. 180, 720-730 (2009).

15. Trompette, A. et al. Allergenicity resulting from functional mimicry of a Toll-like receptor complex protein. Nature 457, 585-588 (2009).

16. Hollingsworth, J.W. et al. The critical role of hematopoietic cells in lipopolysaccharide-induced airway inflammation. Am. J. Respir. Crit. Care Med. 171, 806-813 (2005).

17. Hammad, H., Chieppa, M., Perros, F., Willart, M.A., Germain, R.N. \& Lambrecht, B.N. House dust mite allergen induces asthma via Toll-like receptor 4 triggering of airway structural cells. Nat. Med. 15, 410-416 (2009).

18. Tan, A.M., Chen, H.C., Pochard, P., Eisenbarth, S.C., Herrick, C.A. \& Bottomly, H.K. TLR4 signaling in stromal cells is critical for the initiation of allergic Th2 responses to inhaled antigen. J. Immunol. 184, 3535-3544 (2010).

19. Hahn, I., Klaus, A., Maus, R., Christman, J.W., Welte, T. \& Maus, U.A. Dendritic cell depletion and repopulation in the lung after irradiation and bone marrow transplantation in mice. Am. J. Respir. Cell Mol. Biol. 45, 534-541 (2011).

20. Citrin, D.E. et al. Role of type II pneumocyte senescence in radiationinduced lung fibrosis. J. Natl. Cancer Inst. 105, 1474-1484 (2013).

21. Harfe, B.D., Scherz, P.J., Nissim, S., Tian, H., McMahon, A.P. \& Tabin, C.J. Evidence for an expansion-based temporal Shh gradient in specifying vertebrate digit identities. Cell 118, 517-528 (2004).

22. Bitgood, M.J. \& McMahon, A.P. Hedgehog and Bmp genes are coexpressed at many diverse sites of cell-cell interaction in the mouse embryo. Dev. Biol. 172, 126-138 (1995).

23. Harris, K.S., Zhang, Z., McManus, M.T., Harfe, B.D. \& Sun, X. Dicer function is essential for lung epithelium morphogenesis. Proc. Natl. Acad. Sci. USA 103, 2208-2213 (2006).

24. Morrisey, E.E. \& Hogan, B.L. Preparing for the first breath: genetic and cellular mechanisms in lung development. Dev. Cell 18, 8-23 (2010)

25. de Boer, J. et al. Transgenic mice with hematopoietic and lymphoid specific expression of Cre. Eur. J. Immunol. 33, 314-325 (2003).

26. Jiang, D. et al. Regulation of lung injury and repair by Toll-like receptors and hyaluronan. Nat. Med. 11, 1173-1179 (2005).

27. Bartemes, K.R. \& Kita, H. Dynamic role of epithelium-derived cytokines in asthma. Clin. Immunol. 143, 222-235 (2012).

28. Fort, M.M. et al. IL-25 induces IL-4, IL-5, and IL-13 and Th2-associated pathologies in vivo. Immunity 15, 985-995 (2001).

29. Tamachi, T. et al. IL-25 enhances allergic airway inflammation by amplifying a TH2 cell-dependent pathway in mice. J. Allergy Clin. Immunol. 118, 606-614 (2006). 
30. Ballantyne, S.J. et al. Blocking IL-25 prevents airway hyperresponsiveness in allergic asthma. J. Allergy Clin. Immunol. 120, 1324-1331 (2007).

31. Kurowska-Stolarska, M. et al. IL-33 induces antigen-specific IL-5 + Tcells and promotes allergic-induced airway inflammation independent of IL-4. J. Immunol. 181, 4780-4790 (2008).

32. Ramaprakash, $\mathrm{H}$. et al. Targeting ST2L potentiates CpG-mediated therapeutic effects in a chronic fungal asthma model. Am. J. Pathol. 179, 104-115 (2011).

33. Whitehead, G.S., Thomas, S.Y. \& Cook, D.N. Modulation of distinct asthmatic phenotypes in mice by dose-dependent inhalation of microbial products. Environ. Health Perspect. 122, 34-42 (2013).

34. Fogli, L.K. et al. T cell-derived IL-17 mediates epithelial changes in the airway and drives pulmonary neutrophilia. J. Immunol. 191, 3100-3111 (2013).

35. Miyamoto, M., Prause, O., Sjostrand, M., Laan, M., Lotvall, J. \& Linden, A. Endogenous IL-17 as a mediator of neutrophil recruitment caused by endotoxin exposure in mouse airways. J. Immunol. 170, 4665-4672 (2003).

36. Liang, S.C. et al. An IL-17F/A heterodimer protein is produced by mouse Th17 cells and induces airway neutrophil recruitment. J. Immunol. 179, 7791-7799 (2007).

37. Upham, J.W. \& Stick, S.M. Interactions between airway epithelial cells and dendritic cells: implications for the regulation of airway inflammation. Curr. Drug Targets 7, 541-545 (2006).
38. Lambrecht, B.N. \& Hammad, H. The role of dendritic and epithelial cells as master regulators of allergic airway inflammation. Lancet $\mathbf{3 7 6}, 835-843$ (2010).

39. Guo, L. et al. IL-1 family members and STAT activators induce cytokine production by Th2, Th17, and Th1 cells. Proc. Natl. Acad. Sci. USA 106, 13463-13468 (2009).

40. Angkasekwinai, P. et al. Interleukin 25 promotes the initiation of proallergic type 2 responses. J. Exp. Med. 204, 1509-1517 (2007).

41. Wills-Karp, M. et al. Interleukin-13: central mediator of allergic asthma. Science 282, 2258-2261 (1998).

42. Finkelman, F., Morris, S., Orekhova, T. \& Sehy, D. The in vivo cytokine capture assay for measurement of cytokine production in the mouse. Curr. Protoc. Immunol., Chapter 6, Unit 6.28 (2003).

43. Lewkowich, I.P. et al. PD-L2 modulates asthma severity by directly decreasing dendritic cell IL-12 production. Mucosal Immunol. 6, 728-739 (2013).

44. Divanovic, S. et al. Negative regulation of Toll-like receptor 4 signaling by the Toll-like receptor homolog RP105. Nat. Immunol. 6, 571-578 (2005).

45. Harley, I.T. et al. Differential colonization with segmented filamentous bacteria and Lactobacillus murinus do not drive divergent development of diet-induced obesity in C57BL/6 mice. Mol. Metabol. 2 171-183 (2013). 